\title{
Temporal studies on GRB light curves for neutrino flux predic- tions in multi-collision zone models
}

\author{
Angela Zegarelli ${ }^{1,2, a}$ \\ ${ }^{1}$ Dipartimento di Fisica, Università La Sapienza, P.le Aldo Moro 2, 00185 Roma, Italy \\ ${ }^{2}$ INFN - Sezione di Roma, P.le Aldo Moro 2, 00185 Roma, Italy
}

\begin{abstract}
For the analysis here presented, the most luminous GRB detected by KonusWIND until now was selected, namely GRB 110918A. Through the NeuCosmA code, simulating multi-zone collisions during the GRB prompt emission, a synthetic light curve representative of the observed one has been obtained, in order to study how characteristic parameters of the inner engine activity influence the light curve. This is achieved by varying the input parameters values in the simulation. In agreement with other previous studies, the inner engine is found to be more variable than the observed light curve. Furthermore, the Empirical Mode Decomposition method is applied and its stability is analyzed. Finally, the first multi-collision neutrino flux estimate for a real GRB is presented. Assuming the flux evaluated through the simulation reproducing the GRB gamma-ray light curve and taking into account the data taking conditions and the detector response during the GRB episode, the number of expected signal in the ANTARES neutrino telescope from GRB 110918A was calculated to be $\mu_{s}=6.1 \times 10^{-4}$, which is found to be a factor 20 lower than previous estimations with one-zone models.
\end{abstract}

\section{Introduction}

Gamma-Ray Bursts are the most powerful explosions in the Universe. They constitute highly beamed sources of gamma rays and possibly, in case of hadronic acceleration, also of high-energy neutrinos. In the internal shock scenario, blobs of plasma emitted from a central engine are believed to collide within a relativistic jet and form shocks, responsible for the particle acceleration. Searching for a temporal and spatial coincidence among the GRB prompt emission and high-energy neutrinos is crucial to provide information about the acceleration mechanism in GRBs. The detection of neutrino events would allow to identify these sources as hadronic accelerators and as candidates for Ultra High Energy Cosmic Rays (UHECRs). However, an open issue regarding GRBs is represented by the way in which the central engine operates. In order to shed light on this topic, the information on the temporal behavior of individual GRBs, as observed in gamma rays, is very precious.

Hence, after a brief description in Section 2 of the GRB considered in this work, the simulation model used to reproduce its photon light curve is explained in Section 3. In Section 4, a novel approach for GRB timing studies, which adopts the Hilbert-Huang transform of the light curve for the identification of the characteristic signal modes, is analyzed. However, this method is not found to be stable with

ae-mail: Angela.Zegarelli@roma1.infn.it 
respect to Poissonian fluctuations of the signal. In order to clarify this point, still some investigation is required. Finally, the neutrino flux prediction for this GRB and the number of expected muon neutrino signals in the ANTARES neutrino telescope is presented in Section 5.

\section{Selected candidate: GRB 110918A}

GRB 110918A [1] is the brightest long GRB ever detected by Konus-WIND (KW) and the most luminous one observed until now. At its redshift $(z=0.984 \pm 0.001)$, the high energy fluence measured by $\mathrm{KW}, F_{\gamma}=7.5 \times 10^{-4} \mathrm{erg} \mathrm{cm}^{-2}$, implies a huge isotropic equivalent energy released in the source frame, $E_{\text {iso }}=(2.1 \pm 0.1) \times 10^{54} \mathrm{erg}$, and an isotropic-equivalent peak luminosity $L_{\text {iso }} \sim 4.4 \times 10^{54} \mathrm{erg}$ $\mathrm{s}^{-1}$. The GRB light curve (Figure 1) starts with an extremely bright, hard and short pulse followed by three weaker, softer and partly overlapping pulses in the next $\sim 25 \mathrm{~s}$, showing a long quiescent period $(\sim 10 \mathrm{~s})$ between the second and the third peak. The duration of the burst is $T_{90}=19.6 \pm 0.1$ s. Furthermore, assuming that the shortest timescale in GRB prompt emission (variability time $t_{v}$ ) coincides with the shortest pulse width, the value $t_{v}=0.25 \mathrm{~s}$ was estimated [1].

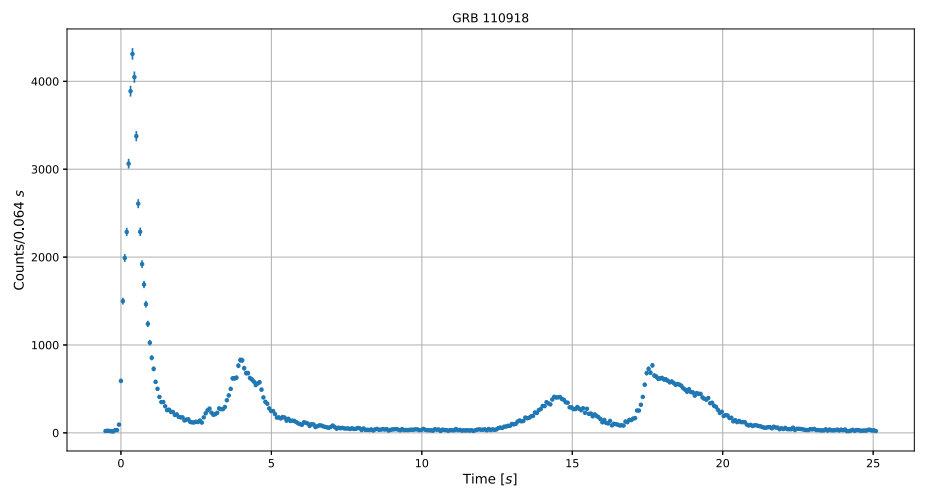

Figure 1: Konus-WIND photon light curve observed from GRB 110918A.

This GRB has been selected because it is ultraluminous, it has peculiar light curve characteristics, and the behavior of its emission is highly non linear, as derived from a temporal study of its Probability Density Function [2]. This latter point allows to use the Hilbert-Huang transformation, as shown in Section 4.

\section{GRB light curve study with multi-collision approach}

In order to simulate a GRB light curve similar to the one of GRB 110918A, the NeuCosmA code [3] has been used. A multi-collision approach [4] allows to simulate collisions at different radii in a GRB jet considering a system of plasma shells with a distribution of Lorentz factors, masses and widths. In this way, an entire light curve is derived and precise secondary flux predictions are obtained, including neutrinos. Therefore, a synthetic GRB light curve reproducing that of GRB 110918A has been constructed. After choosing several input parameters (e.g. radius and Lorentz factor distributions of the shells), 500 simulations have been performed and the averaged light curve has been computed 
(Figure 2). The variability time $t_{\mathrm{v}}=0.220 \pm 0.008 \mathrm{~s}$, the burst duration $T_{90}=17.136 \pm 0.003 \mathrm{~s}$ and the number of collisions $N_{\text {coll }}=78 \pm 3$ for the averaged simulated light curve are consistent with the quantities observed for GRB 110918A: $t_{\mathrm{v}}=0.25 \mathrm{~s}, T_{90}=19.6 \pm 0.1 \mathrm{~s}$ and $N_{\text {coll }} \simeq 79$. As the number of observed pulses $\left(N_{\text {pulses }}=5\right)$ is much smaller than the number of ejected shells $\left(N_{\text {shells }}=110\right)$, the inner engine results more variable than the observed light curve [5].

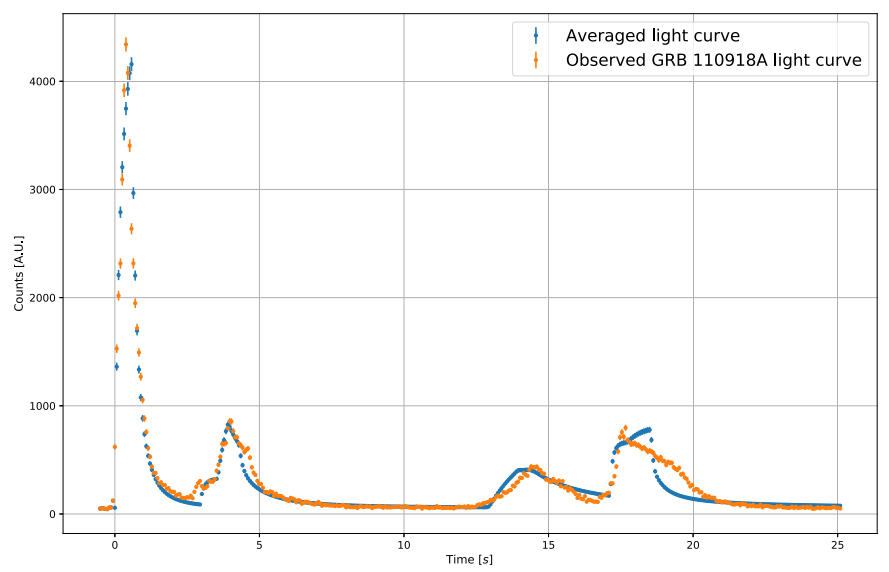

Figure 2: GRB 110918A Konus-WIND observed photon light curve (in orange, same as in Figure 1) and photon light curve simulated with the multi-collision NeuCosmA code (in blue).

\section{Hilbert-Huang transform: a novel approach for GRB timing studies}

Non-linear and non-stationary time series allow for an analysis through the Hilbert-Huang transformation (HHT) [6]. Its use for the GRB timing studies, a powerful tool to understand the physics of the GRB prompt emission and its variability, is a recent idea, given the lack of direct observations of the inner engine. Such an analysis consists of two steps:

1. Empirical Mode Decomposition (EMD): decomposition of the signal into individual modes embedded in the data (Intrinsic Mode Functions, IMFs), that relies on the assumption that data consist of different simple intrinsic modes of oscillation.

2. Hilbert Spectral Analysis: the Hilbert transform is applied to each IMF in order to construct the energy-frequency-time distribution, called Hilbert spectrum, in which the time localities of events is preserved.

The stability of this method has been investigated: while translation and addition of a constant factor to the light curve do not influence results in individual IMFs, the addition of random fluctuations produces relevant modification to the EMD. For this reason, it was not possible to compare the results of HHT obtained with the simulated and with the observed light curve. 


\section{Neutrino flux prediction}

In this work the first multi-collision neutrino flux estimate for a real GRB is presented (Figure 3). Assuming the neutrino flux evaluated through the simulation reproducing the GRB light curve and taking into account the data taking conditions and the detector response during the GRB episode [7], the number of expected signal in the ANTARES neutrino telescope from GRB 110918A was calculated: $\mu_{s}=6.1 \times 10^{-4}$. This value is smaller than the one previously calculated by the ANTARES Collaboration according to the one-zone approach, namely $\mu_{s}=1.3 \times 10^{-2}$ [8]. This is connected with the fact that the novel modeling presented here accounts for the whole inner engine behavior, as dictated by the emitted light curve, while past calculations simplified such a description within a unique collision able to reproduce the gamma-ray spectrum. The limited expected event rate justifies the lack of spatial and temporal coincidence in ANTARES between high-energy neutrinos and GRB events until now [9][10].

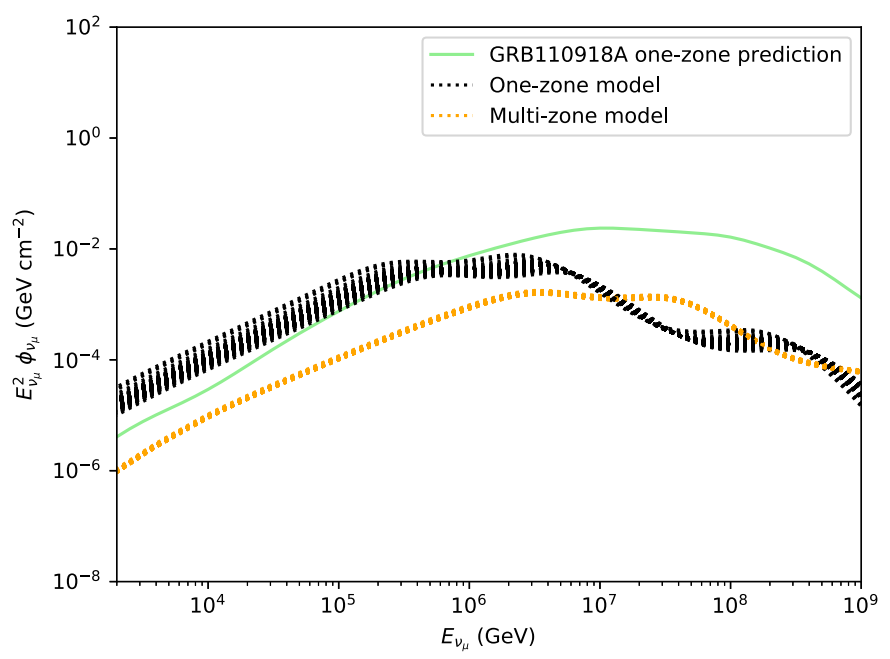

Figure 3: Expected $v_{\mu}+\bar{v}_{\mu}$ fluence for the GRB 110918A. Green solid line: former one-zone prediction, extracted from the gamma-ray spectrum [8]. Dashed black lines: one-zone prediction obtained by averaging over 500 complete light curve simulations performed using the NeuCosmA code (this work). Dashed orange lines: multi-zone prediction obtained by averaging over 500 complete light curve simulations performed using the NeuCosmA code (this work).

\section{References}

[1] D. D. Frederiks, et al., ApJ, 779, 151, 2013

[2] N. Bhatt \& S. Bhattacharyya, MNRAS, 420, 1706, 2012

[3] S. Hümmer, et al., ApJ, 721, 630, 2010

[4] M. Bustamante, et al., Astrophys. J., 837, 33, 2017

[5] E. Nakar \& T. Piran, MNRAS, 331, 40, 2002 
[6] N. E. Huang, et al., Proc. R. Soc. Lond. A, 454, 903, 1998

[7] S. Celli, Proceeding of RICAP16, EPJ Web of Conferences, 136 , 04006, 2017

[8] A. Albert, et al., MNRAS, 469, 906, 2017

[9] S. Adrián-Martínez et al., ANTARES Collaboration, A\&A 559, A9, 2013

[10] S. Celli, Proceeding of ICRC17, Proceedings of Science, 301, 998, 2017 